tive) ring, $H$ a subring of $K$ and that ( $\alpha) H$ has a 1-element; $(\beta)$ the equation $x h=h_{1}$ with $h, h_{1} \in H, x \in K, h \neq 0$ implies that $x \in H,(\gamma)$ for every $a \in K, b \in H$, there exists an element $b_{1}$ in $H$ with $b a=a b_{1}$. If $H \neq K$, it follows that every element of $H$ commutes with every element of $K$.

UNIVERSITY OF TORONTO

\title{
A THEOREM ON INTEGRAL SYMMETRIC MATRICES ${ }^{1}$
}

B. W. JONES

Though the following theorem yields important results in the theory of quadratic forms, its statement and proof are independent of such theory and seem to possess significance in their own right.

THEOREM. Let $A$ and $B$ be symmetric integral nonsingular matrices with respective dimensions $n$ and $m(n>m)$ and $S$ an $n$ by $m$ matrix of rank $m$ with rational elements such that $s$ is the 1.c.m. of the denominators and $S^{T} A S=B$. Then there is an $n$ by $n$ matrix $T$ with rational elements the prime factors of whose denominators all divide $s$, whose determinant is 1 and which takes $A$ into an integral matrix $A_{0}$ which represents $B$ integrally, that is, $U^{T} A_{0} U=B$ for some integral matrix $U$.

To prove this we first, for brevity's sake, define an s-matrix or $s$-transformation to be one with rational elements the prime factors of whose denominators all divide $s$. Then write $R=s S$, and, by elementary divisor theory, determine unimodular matrices $P$ and $Q$ such that

$$
P R Q=\left[\begin{array}{c}
R_{1} \\
0
\end{array}\right]=s\left[\begin{array}{c}
S_{1} \\
0
\end{array}\right]=s S^{\prime}
$$

where $R_{1}$ is the diagonal matrix $r_{1} \dot{+} r_{2} \dot{+} \cdots \dot{+} r_{m}, \dot{+}$ denotes direct sum, $r_{i}$ divides $r_{i+1}$ for $i=1,2, \cdots, m-1$ and $S^{\prime}$ and $S_{1}$ are defined by the equations. Write $r_{i} / s=u_{i} / s_{i}$ where the latter fraction is in lowest terms and $s_{i}>0$. Then $s_{i}$ is divisible by $s_{i+1}$ and hence $s_{i}$ is prime to $u_{j}$ for $j \leqq i$.

\footnotetext{
Presented to the Society, September 10, 1948; received by the editors June 7, 1948.

${ }^{1}$ This paper was written while on sabbatical leave from Cornell University with the aid of a grant from the Research Corporation.
} 
If we write $A^{\prime}=P^{I T} A P^{I}$ and $B^{\prime}=Q^{T} B Q$, the equality $S^{T} A S=B$ becomes $S^{\prime T} A^{\prime} S^{\prime}=B^{\prime}$ which, with $A^{\prime}=\left(a_{i j}\right)$ and $B^{\prime}=\left(b_{i j}\right)$, implies

$$
a_{i j} r_{i} r_{j} / s^{2}=a_{i j} u_{i} u_{j} / s_{i} s_{j}=b_{i j}, \quad i, j=1,2, \cdots, m .
$$

Suppose $s_{i}=1=\left|u_{i}\right|$ for $i=1,2, \cdots, h-1$ but not for $i=h$. Then (1) and $B^{\prime}$ integral implies that $s_{h}$ divides $a_{i h}$ for $1 \leqq i \leqq h$ and $s_{h}^{2}$ divides $a_{h h}$. Moreover $u_{h}$ divides $b_{i h}$ for $1 \leqq i \leqq m$ and $u_{h}^{2}$ divides $b_{h h}$. Write $A^{\prime}$ in the form

$$
\left[\begin{array}{cc}
A_{11} & A_{12} \\
A_{12}^{T} & A_{22}
\end{array}\right]
$$

where $A_{11}$ is an $h$ by $h$ matrix. Let $D_{h}$ be the matrix obtained from the $h$-rowed identity matrix by replacing its last diagonal element by $s_{h}$ and $U_{h}$ the matrix obtained from the $m$-rowed identity matrix by replacing its $h$ th diagonal element by $u_{h}$. Write $F_{h}=D_{h}^{I}+K_{h}$ where $K_{h}$ is an integral $(n-h)$-rowed square matrix of determinant $s_{h}$ later to be determined. We then have

$$
F_{h}^{T} A^{\prime} F_{h}=\left[\begin{array}{cc}
D_{h}^{I} A_{11} D_{h}^{I} & D_{h}^{I} A_{12} K_{h} \\
K_{h}^{T} A_{12}^{T} D_{h}^{I} & K_{h}^{T} A_{22} K_{h}
\end{array}\right]=A_{h} .
$$

Then $S_{h}$, defined by the equation $S_{h}=F_{h}^{I} S^{\prime} U_{h}^{I}$, is the direct sum of the integral matrix $u_{1} \dot{+} u_{2} \dot{+} \cdots+u_{h-1} \dot{+} 1$, whose elements have absolute value 1 and an $n-h$ by $m-h s$-matrix. Furthermore $S^{\prime} A^{\prime} S^{\prime}=B^{\prime}$ becomes $S_{h}^{T} A_{h} S_{h}=B_{h}$ where the equality $U_{h}^{I} B^{\prime} U_{h}^{I}=B_{h}$ defines $B_{h}$. Now $B_{h}$ is integral since, as shown above, $u_{h}$ divides $b_{i h}$ for $1 \leqq i \leqq m$ and $u_{h}^{2}$ divides $b_{h h}$. Furthermore $B_{h}$ represents $B^{\prime}$ integrally. Similarly $D_{h}^{I} A_{11} D_{h}^{I}$ is integral.

Next we determine $K_{h}$ consistent with the above conditions so that $A_{h}$ is integral, that is, so that $D_{h}^{I} A_{12} K_{h}$ is integral. In fact, in view of the definition of $D_{h}$, we need only make $\alpha K_{h}$ divisible by $s_{h}$ where $\alpha$ is the $h$ th row of $A_{12}$. This is easily done by finding a unimodular matrix $W$ so that $\alpha W \equiv(w, 0, \cdots, 0)\left(\bmod s_{h}\right)$ and choosing $W^{I} K_{h}$ to be the diagonal matrix $s_{h} \dot{+}+\cdots+1$ of determinant $s_{h}$.

If now we diagonalize the last $n-h$ rows of $S_{h}$ replacing $A_{h}$ and $B_{h}$ by equivalent matrices (that is, matrices obtained from them by unimodular transformations) we may continue along the above lines to derive inductively a sequence of integral matrices $A_{h}$ obtained from $A$ by $s$-transformations of determinant 1 and taken by transformations $S_{h}$ into integral matrices $B_{h}$ which represent $B^{\prime}$ integrally, each $S_{h}$ being the direct sum of an $h$-rowed integral matrix and an $s$-matrix. 
Then $S_{m}$ is integral and taking $A_{m}=A_{0}, B_{m}=B_{0}$ we see that $A_{0}$ is obtained from $A$ by an $s$-transformation of determinant 1, represents $B_{0}$ integrally and hence $B$ integrally.

Since two quadratic forms with integral coefficients may be defined to be in the same genus if one may be taken into the other by a transformation of determinant \pm 1 with rational elements whose denominators are prime to twice the determinant of the form, we have the following

COROLLARY 1. If $f$ and $g$ are two quadratic forms with integral coeffcients, having nonzero determinants and $n$ and $m$ variables respectively, $n>m$, and if there is an $n$ by $m$ matrix with rational elements whose denominators are prime to $2|f|$ and taking $f$ into $g$, then there is a form $f_{0}$ in the same genus as $f$ whose matrix has integral elements and which represents $g$ integrally.

This corollary has been proved using the theory of quadratic forms by C. L. Siegel, ${ }^{2}$ whose proof contained some ideas in common with the above proof, by Hel Braun ${ }^{3}$ and probably follows from the much earlier work of Minkowski. However in all these sources the condition that the denominators be prime to $2|f|$ is essential. It is then of interest to note that this restriction is not necessary for the theorem of this note.

In fact, a direct consequence of our theorem (as noted by the referee) is

CoRollary 2. If a classic quadratic form $f$ (that is, a form whose matrix has integer elements) represents an integer or classic form $\mathrm{g}$ rationally, then some classic form $f^{\prime}$ of the same determinant as $f$ represents $g$ integrally.

Thus for example the conditions for integral representation by the form $x_{1}^{2}+x_{2}^{2}+\cdots+x_{n}^{2}, 1 \leqq n \leqq 7$, are the same conditions as those for rational representation since there is only one class of forms of determinant 1 in these cases.

Cornell University

2 C. L. Siegel, Über die analytische Theorie der quadratischen Formen, Ann. of Math. (2) vol. 36 (1935) pp. 527-606, Lemma 24.

${ }^{3}$ Hel Braun, Geschlechter quadratischer Formen, J. Reine Angew. Math. vol. 182 (1940) pp. 32-49. 